\title{
Studying an Agulhas ring's long-term pathway and decay with finite-time coherent sets
}

\author{
Gary Froyland, ${ }^{1}$ Christian Horenkamp, ${ }^{2}$ Vincent Rossi, ${ }^{3}$ and Erik van Sebille ${ }^{4,5}$ \\ ${ }^{1}$ School of Mathematics and Statistics, University of New South Wales, Sydney, New South Wales 2052, \\ Australia \\ ${ }^{2}$ Department of Mathematics, University of Paderborn, 33098 Paderborn, Germany \\ ${ }^{3}$ IFISC (Institute for Cross-Disciplinary Physics and Complex Systems), CSIC-UIB, Palma de Mallorca 07122, \\ Spain \\ ${ }^{4}$ Climate Change Research Centre and ARC Centre of Excellence for Climate System Science, \\ University of New South Wales, Sydney, New South Wales 2052, Australia \\ ${ }^{5}$ Grantham Institute and Department of Physics, Imperial College London, London SW7 2AZ, United Kingdom
}

(Received 18 December 2014; accepted 23 July 2015; published online 27 August 2015)

Coherent sets in dynamical systems are regions in phase space that optimally "carry mass" with them under the system's evolution, so that these regions experience minimal leakage. The dominant tool for determining coherent sets is the transfer operator, which provides a complete description of Lagrangian mass transport. In this work, we combine existing transfer operator methods with a windowing scheme to study the spatial and temporal evolution of a so-called Agulhas ring: a large anticyclonic mesoscale eddy playing a key role in inter-ocean exchange of climate-relevant properties. Our focus is on ring decay over time and the windowing scheme enables us to study how the most coherent region (our estimate of the ring) varies in position and size over a period of more than two years. We compare the eddy-like structure and its spatio-temporal changes as revealed by our method and by a classical Eulerian approach. (C) 2015 AIP Publishing LLC.

[http://dx.doi.org/10.1063/1.4927830]

Finite-time coherent sets are determined by assembling gridded Lagrangian trajectories into a grid-to-grid transition matrix and computing dominant singular vectors of this matrix. We introduce a new combination of transfer operator based coherent set techniques with an overlapping window scheme, which enables ring size to vary over time, and follow an Agulhas ring's west-north-westward pathway across the southern Atlantic Ocean for over more than 2 years. We describe the evolution of the ring during this long journey and draw relationships between various dynamical and physical quantities associated with the ring and its decay. The advantage of the transfer operator method is that mass loss from rings is explicitly minimised over moderate periods of time using Lagrangian trajectory information. Our overlapping window approach readily accommodates the decay of rings over longer timespans during which part of the fluid is expelled from the structure. The ring boundary obtained by our method can then be used to explicitly study ring decay in both Eulerian and Lagrangian senses.

\section{INTRODUCTION}

The study of transport and mixing processes in timedependent flows is relevant for a variety of natural phenomena, such as biological dynamics, astrodynamics, molecular dynamics, and fluid dynamics, including those of the ocean and the atmosphere. The classical dynamical systems approach to transport in the autonomous or periodic time-dependent case has revolved around invariant manifolds, which can represent codimension one transport barriers. If trajectories locally experience (possibly nonuniform) asymptotic expansion and contraction, and the vector field is sufficiently smooth, then local stable and unstable manifolds exist. ${ }^{25,26}$ Each of these manifolds is a material or equivariant object and is exactly evolved to their counterparts at later times. The traditional focus has been on those invariant manifolds that correspond to equilibria or periodic orbits of low period. The reasoning behind this is that these fixed and low-period structures are important in organising the dynamics. This approach has been popularised in so-called "lobe dynamics," ${ }^{28}$ which provides a mechanism by which transport can occur across curves formed by the unions of segments of different invariant manifolds when there is periodic driving. However, in the setting with aperiodic time-dependence, there are often no equilibria that remain fixed in space throughout one driving period, nor periodic orbits. Thus, it is crucial to identify the most important material curves or surfaces. There are a number of methods that have been proposed to select distinguished curves. These include Lagrangian and complexity descriptors, such as ${ }^{22,29}$ finite-time Lyapunov exponents, ${ }^{31}$ which look for maximally stretching local curves, which in 2D hyperbolic area-preserving flows are associated with curves of maximal local repulsion and low cross-transport, over a finite horizon. Other methods compare local stretching or shearing with nearby curves, ${ }^{19}$ and attempt to find curves with minimal local deformation, again over a finitetime horizon. The rationale is that diffusive transport across a boundary is favored by increased filamentation of that boundary. 
The approach taken in the present paper is to directly (as opposed to inferring their presence from codimension one transport barriers) identify regions in the phase space that are maximally coherent, i.e., that are the best transporters of mass with minimal leakage over a finite time duration. The method used to find such regions is based on transfer operators, or in practice, transition matrices of a coarse-graining of the state space. The basic methodology originates from Ref. 17, in which the term "finite-time coherent sets" is coined in this transport context. We have used this approach in a previous study ${ }^{15}$ to analyse a single Agulhas ring in three-dimensions based on model data over a short time period of 6 months. In contrast, here we track an Agulhas ring over 2 years based on two-dimensional satellite-derived velocity field. Long time-scales are commonly considered in geophysics, especially for many crucial climate-relevant issues, because the coupled ocean-atmosphere system is characterized by interacting processes occurring at multiple scales ranging from submeso- to global-scale.

Recent work ${ }^{14}$ provides an analytic setup, proving various optimality results in a mesh-free setting. The approach in Refs. 14 and 17 focusses on determining a pair of coherent sets: one at a specified initial time and the other at a specified final time. It has been observed in Ref. 16 that in the presence of strong hyperbolicity, the coherent set at initial time has boundaries mostly aligned with stable directions, while the coherent set at the final time has boundaries mostly aligned with unstable directions. This is a quite natural result as the method of Refs. 14 and 17 tries to minimise diffusive leakage across the boundaries of the coherent sets by reducing their boundary sizes at both initial and final times. If one wishes to link independent coherent set calculations on adjacent time windows, it is preferable to consider "unbiased" (neither stable nor unstable) coherent sets. Two modifications are proposed in Ref. 16: one involves the computation of a related matrix and is considered in detail in Ref. 16, while the other reuses information from the original computations and is only briefly mentioned in Section 5.2 of Ref. 16. It is this latter approach that we develop and further explore here, motivated by long-term tracking of Agulhas rings. We choose the latter approach as it is less memoryintensive (the matrices used in the computation are much sparser) and one has more control on the diffusion process.

From an oceanographic point of view, we wish to quantify the amount of water effectively transported by oceanic eddies. This is tantamount to assessing the "leakage" of water occurring across the boundaries of the structure. Agulhas rings, anti-cyclonically rotating vortices with a typical diameter of $200 \mathrm{~km}$, are among the largest meso-scale eddies in the ocean and form south of Africa due to instabilities in the Agulhas Current and its retroflection, e.g., Refs. 1 and 9. As the Agulhas rings shed from the main current pathway, they start moving north-westward in the South Atlantic due to the latitude dependence of the Coriolis parameter. ${ }^{34}$ It has long been thought that the five to six rings per year are the dominant agents by which Indian Ocean water enters the Atlantic Ocean. ${ }^{18}$

Recently, ${ }^{2}$ tracked Agulhas rings using geodesic theory, focussing on the coherent, non-leaking, portion of the rings.
They look for curves that enclose areas with very little change over time. This curve is usually in the interior of the Agulhas ring, where the dynamics is more regular and spatially separated from more complicated dynamics occurring further from the ring's core. However, both Refs. 12 and 35 suggested that at least half of the transport of water from the Indian to the Atlantic Ocean occurs outside of Agulhas rings through horizontal advection mediated by (sub)mesoscale filaments and jets. Using satellite data, Refs. 30 and 5 have also shown that Agulhas rings reduce in surface area as they travel across the South Atlantic Ocean. This crucial process for climatic issues, referred to as "ring decay," has been associated to a significant leaking of the warm and salty Indian Ocean waters within the core of the eddy into the colder and fresher South Atlantic Ocean surrounding waters. Indeed, while some authors [e.g., Ref. 2] focused their efforts on tracking the cores of Agulhas rings, it is more relevant for climatic questions to examine how much water an Agulhas ring loses along its path across the Atlantic Ocean. To do this, it is necessary to properly define 3-dimensional eddylike structures with the objective detection of their boundaries. ${ }^{3,15}$ However, since 3-dimensional oceanic datasets rely essentially on numerical models, other authors intend to derive surface proxies of the "ring decay.",10,30,32,33

Here, we use data from satellite altimetry to focus on a typical Agulhas ring (shed in December 1998 from the Agulhas Current), using it as a case study to test our novel eddy-detection technique and investigate its decay. This paper is organised as follows. In Section II, we introduce a method for the tracking of coherent sets and allowing mass loss over time, based on several independent transfer operator calculations. This method is applied in Section III to the oceanic surface flow and the results are discussed in Section IV to conclude in Section IV C.

\section{TRACKING THE EVOLUTION OF COHERENT SETS}

\section{A. A pair of coherent sets}

In this study, we make repeated use of the concept of coherent pairs, introduced by Froyland et al. ${ }^{17}$ to create a sequence of several dynamically connected coherent sets. We consider the following time-dependent ordinary differential equation (ODE):

$$
\dot{x}=f(x, t),
$$

on $\mathcal{X} \subset \mathbb{R}^{d}$, with $f: \mathcal{X} \times \mathbb{R} \rightarrow \mathbb{R}^{d}$ sufficiently smooth that for every initial condition $x\left(t_{0}\right)=x_{0}$, a unique solution $x$ : $\mathbb{R}_{+} \rightarrow \mathcal{X}$ exists. Then, $\phi: \mathbb{R} \times \mathbb{R}_{+} \times \mathcal{X} \rightarrow \mathcal{X}$ with $\phi\left(t_{0}, \tau, x_{0}\right)=x(\tau)$, defines the flow generated by the ODE (1).

In the following, we restrict to a subset $X$ of the domain $\mathcal{X}$, where we seek pairs of sets for which the probability of a trajectory flowing from the initial to the final set over a specific time interval is maximized. For this reason, in the remainder of the paper, we denote by $t \in \mathbb{R}$ the initial point in time and by $t+\tau$, with $\tau \in \mathbb{R}_{+}$, the final point in time. Thus, the pair $A_{t}, A_{t+\tau}$ of subsets of $X$ should satisfy $A_{t+\tau} \approx \phi\left(t, \tau, A_{t}\right)$. To measure the likelihood of points flowing from $A_{t}$ to $A_{t+\tau}$, we denote by $m_{X}$ the normalized 
Lebesgue (volume) measure on $X$. As our focus here is on seawater, whose density can be approximated to be quasiuniform, we use volume as a proxy for water mass and compute the amount of mass which is transported from $A_{t}$ to $A_{t+\tau}$ over the time $\tau$

$$
\rho\left(A_{t}, A_{t+\tau}\right):=\frac{m_{X}\left(A_{t} \cap \phi\left(t+\tau,-\tau, A_{t+\tau}\right)\right)}{m_{X}\left(A_{t}\right)} .
$$

We call $\rho\left(A_{t}, A_{t+\tau}\right)$ for a fixed $t$ and $\tau$ the coherence of the pair $A_{t}, A_{t+\tau}$ over the duration $\tau$.

The numerical methodology proposed in Ref. 17 finds pairs of sets for which the coherence is maximized under the addition of small-amplitude diffusion (this idea is formalised in Ref. 14). The first step is to cover $X$ and the image $Y=$ $\phi(t, \tau, X) \subset \mathcal{X}$ by a collection of disjoint subsets of small diameter, $\left\{B_{1}, \ldots, B_{m}\right\}$ and $\left\{C_{1}, \ldots, C_{n}\right\}$, respectively. To obtain probabilistic information on trajectories flowing from one subset of $X$ to another, we construct the transition matrix

$$
\mathbf{P}_{t, \tau ; i, j}:=\frac{m_{X}\left(B_{i} \cap \phi\left(t+\tau,-\tau, C_{j}\right)\right)}{m_{X}\left(B_{i}\right)} .
$$

The entry $\mathbf{P}_{t, \tau ; i, j}$ can be interpreted as the probability that a particle selected uniformly at random in $B_{i}$ at time $t$ will be in $C_{j}$ at time $t+\tau$. The matrix (3) is a finite-dimensional approximation of the $\phi(t, \tau, \cdot)$-induced action on signed probability measures.

The entries $\mathbf{P}_{t, \tau ; i, j}$ of (3) cannot be easily calculated exactly and are typically numerically approximated: choose $l$ test points $\left\{z_{i, r}\right\}_{r=1 \ldots l}$ uniformly distributed over each box $B_{i}$, $i=1, \ldots, m$ and compute

$$
\tilde{\mathbf{P}}_{t, \tau ; i, j}=\frac{\sharp\left\{r: z_{i, r} \in B_{i}, \phi\left(t, \tau, z_{i, r}\right) \in C_{j}\right\}}{l} .
$$

Under the assumptions on $\phi$, for fixed $t$ and $\tau$, $\left|\tilde{\mathbf{P}}_{t, \tau ; i, j}-\mathbf{P}_{t, \tau ; i, j}\right| \rightarrow 0$ for each $i, j$ as $l \rightarrow \infty$. In general, $l$ should be chosen so that $\left\{\phi\left(t, \tau, z_{i, r}\right): r=1, \ldots, l\right\}$ is a good representation of $\phi\left(t, \tau, B_{i}\right)$ for each $i=1, \ldots, m$. For the remainder of the paper, we revert to the notation $\mathbf{P}$ with the understanding that numerically we use $\tilde{\mathbf{P}}$.

We set $p_{i}=m_{X}\left(B_{i}\right)$ for $i=1, \ldots, m$ and define $q:=p \mathbf{P}_{t, \tau}$. The vector $q$ denotes the distribution of mass on the $C_{j}, j=1, \ldots, n$, after applying the dynamics given by $\phi$ from time $t$ to $t+\tau$. The following algorithm ${ }^{17}$ describes how to identify the most coherent pair of sets, denoted $\hat{A}_{t}, \hat{A}_{t+\tau}$, using singular vectors of P. Steps 1-3 perform the singular vector computation, and steps 4-6 threshold the left and right singular vectors and ensure that the masses of the two coherent sets $A_{t}, A_{t+\tau}$ are as similar as possible, considering a given box resolution.

Algorithm 1 (Input: $p, \mathbf{P}$; Output: $u_{2}, v_{2}, \hat{b}, \hat{b}^{\prime}, \rho, \hat{A}_{t}, \hat{A}_{t+\tau}$ )

(1) Define diagonal matrices $\left(\Pi_{p}\right)_{i i}=p_{i}, i=1, \ldots, m$, and $\left(\Pi_{q}\right)_{j j}=q_{j}, j=1, \ldots, n$.

(2) Compute the second largest singular value $\sigma_{2}<1$ of $\Pi_{p}^{1 / 2} \mathbf{P} \Pi_{q}^{-1 / 2}$ and corresponding left and right singular vectors $\tilde{u}_{2}, \tilde{v}_{2}$.

(3) Set $u_{2}:=\tilde{u}_{2} \Pi_{p}^{-1 / 2}, v_{2}:=\tilde{v}_{2} \Pi_{q}^{-1 / 2}$.
(4) Denote $I(b)=\left\{i: u_{2, i}>b\right\}, J\left(b^{\prime}\right)=\left\{j: v_{2, j}>b^{\prime}\right\}$. Perform a line search on $b$ to solve

$$
\rho:=\max _{b \in \mathbb{R}: \sum_{i \in I(b)} p_{i} \leq 1 / 2} \frac{\sum_{i \in I(b), j \in J\left(b^{\prime}\right)} p_{i} \mathbf{P}_{i j}}{\sum_{i \in I(b)} p_{i}}
$$

selecting $b^{\prime}=b^{\prime}(b)$ so that $\left|\sum_{i \in I(b)} p_{i}-\sum_{j \in J\left(b^{\prime}\right)} q_{j}\right|$ is minimised.

(5) Repeat step 4, replacing $u_{2}$ with $-u_{2}$ and $v_{2}$ with $-v_{2}$ (note this is equivalent to redefining $I(b)=\left\{i: u_{2, i}<\right.$ $b\}, J\left(b^{\prime}\right)=\left\{j: v_{2, j}<b^{\prime}\right\}$ to consider sublevel sets rather than superlevel sets). In step 6 (and for the Output of this Algorithm), use the parity that yields the maximum value of $\rho$.

(6) Denote by $\hat{b}, \hat{b}^{\prime}$ the optimal $b, b^{\prime}$ and set $\hat{A}_{t}:=\cup_{i \in I(\hat{b})} B_{i}$, $\hat{A}_{t+\tau}:=\cup_{j \in J\left(\hat{b}^{\prime}\right)} C_{j}$.

For applications in which an a priori known structure needs to be identified, the selection of the parity in step 5 can be replaced in such a way that the positive entries of $u_{2}$ and $v_{2}$ indicate the desired structure. For example, if we wish to identify a ring, either the positive entries of $u_{2}$ and $v_{2}$ indicate a ring or they indicate the rest of the domain with a "hole" where the ring is; we choose the parity of $u_{2}$ and $v_{2}$ to indicate the former. In Section IV, we will use this modification to identify a sequence of Agulhas rings along the ring's pathway in the South Atlantic Ocean.

\section{B. Computing a sequence of coherent sets via overlapping time windows}

The output of Algorithm 2 is designed to maximize the amount of mass that is transported from the initial set $A_{t}$ at time $t$ to the final set $A_{t+\tau}$ at time $t+\tau$ in the presence of small diffusion. Furthermore, by construction, the surface areas (captured by $p$ and $q$ at times $t$ and $t+\tau$, respectively) of $A_{t}$ and $A_{t+\tau}$ are (very close to) identical. In the case of divergent flows (e.g., when Ekman currents are added to the geostrophic currents), the vector $p$ would describe surface water mass rather than surface area. In the above specific example, we would replace the definition $p_{i}=m_{X}\left(B_{i}\right)$ with $p_{i}=\int_{B_{i}} \varrho(x) d m(x)$, where $\varrho: X \rightarrow \mathbb{R}^{+}$is the twodimensional density of seawater and $m$ is two-dimensional Lebesgue measure (area). The vector $q$ is defined as before: $q=p \mathbf{P}_{t, \tau}$. Such a divergent situation was studied in two and three dimensions in Example 2 of Ref. 17 for atmospheric flows.

To calculate intermediate coherent structures, one can use the push forward of the initial set $A_{t}$ over a time shorter than $\tau$. However, this approach is not useful for estimating the decay of Agulhas rings over very long flow durations because we expect the surface area of these rings to shrink as they eventually decay. Therefore, we present in the following a novel approach allowing a family of coherent sets computed over shorter time durations to shrink or grow over an extended period of time. This is necessary in order to study the rate at which the ring decays.

Thus, given times $t_{1}, \ldots, t_{N}$, we wish to generate a corresponding sequence of maximally coherent sets $\bar{A}_{t_{1}}, \ldots, \bar{A}_{t_{N}}$. We fix $\tau$ to be the time duration over which we wish to 
calculate coherence and consider time windows $\left[t_{1}-\tau, t_{1}+\right.$ $\tau], \quad\left[t_{2}-\tau, t_{2}+\tau\right], \ldots,\left[t_{N}-\tau, t_{N}+\tau\right]$. Because we desire some continuity properties of the $\hat{A}_{t}$ with respect to $t$, these windows need to overlap so that adjacent windows share some dynamics. At a minimum, we require $t_{k+1}-t_{k}<2 \tau$, $k=1, \ldots, N-1$, and better, $t_{k+1}-t_{k} \leq \tau$ so that adjacent windows share at least half of their dynamics. Each time window $\left[t_{k}-\tau, t_{k}+\tau\right]$ will be independently used to calculate a coherent set $A_{t_{k}}, k=1, \ldots, N$.

For each $k=1, \ldots, N$, we build an $m_{k} \times n_{k}$ transition matrix $\mathbf{P}_{t_{k}-\tau, 2 \tau}$ (describing dynamics over $\left[t_{k}-\tau, t_{k}+\tau\right]$ ) and an $m_{k} \times o_{k}$ transition matrix $\mathbf{P}_{t_{k}-\tau, \tau}$ (describing dynamics over $\left.\left[t_{k}-\tau, t_{k}\right]\right)$. The basic idea is to run Algorithm 1 using $\mathbf{P}_{t_{k}-\tau, 2 \tau}$ to obtain a coherent pair over the full time window $\left[t_{k}-\tau, t_{k}+\tau\right]$. The mass of the sets $\hat{A}_{t_{k}-\tau}, \hat{A}_{t_{k}+\tau}$ will fix the mass for the coherent set at time $t_{k}$, but we do not yet have a coherent set at $t_{k}$; only at $t_{k}-\tau$ and $t_{k}+\tau$. To get a coherent set at $t_{k}$, we push forward the vector $u_{2}$ at time $t_{k}-\tau$ to time $t_{k}$ with the second matrix $\mathbf{P}_{t_{k}-\tau, \tau}$ and then threshold to match masses at times $t_{k}-\tau$ and $t_{k}+\tau$. We refer to Figure 1 for a schematic illustration of Algorithm 2.

In the following algorithm, we denote by $p^{k}$ the $1 \times m_{k}$ probability vector, $p_{i}^{k}=m\left(B_{i}^{k}\right) / \sum_{i=1}^{m_{k}} m\left(B_{i}^{k}\right)$, where $m$ still measures volume and $B_{1}^{k}, \ldots, B_{m_{k}}^{k}$ denote boxes covering the relevant part of phase space at time $t_{k}-\tau$. Similarly, the vector $r^{k}$ will describe the volume of the box collection $\left\{D_{1}^{k}, \ldots, D_{o_{k}}^{k}\right\}$ at time $t_{k}$.

Algorithm 2 (Input: $p^{k}, \mathbf{P}_{t_{k}-\tau, \tau}, \mathbf{P}_{t_{k}-\tau, 2 \tau}$; Output: $\bar{A}_{t_{k}}$ )

(1) Apply Algorithm 1 using $p^{k}, \mathbf{P}_{t_{k}-\tau, 2 \tau}$ as input, yielding $u_{2}, v_{2}, \hat{b}, \hat{b}^{\prime}, \hat{A}_{t_{k}-\tau}, \hat{A}_{t_{k}+\tau}$ as output.

(2) Define $r^{k}=p^{k} \mathbf{P}_{t_{k}-\tau, \tau}$ (the mass probability vector at time $t_{k}$ on the box collection $\left.D^{k}\right)$

(3) Set $w_{2}=\left(u_{2} \mathbf{P}_{t_{k}-\tau, \tau}\right) / r^{k}$, where division is meant component-wise (this pushes forward the vector $u_{2}$, which is anchored at time $t_{k}-\tau$ to the vector $w_{2}$, which is anchored at the desired time $\left.t_{k}\right)$.

(4) Denote $H(b)=\left\{h: w_{2, h}>b\right\}$ and select $b^{\prime \prime}$ so that $\left|\sum_{i \in I(\hat{b})} p_{i}^{k}-\sum_{h \in H\left(b^{\prime \prime}\right)} r_{h}^{k}\right|$ is minimised, denoting the minimal $b^{\prime \prime}$ by $\hat{b}^{\prime \prime}$.

(5) Set $\bar{A}_{t_{k}}=\cup_{h \in H\left(\hat{b}^{\prime \prime}\right)} D_{h}^{k}$.

Remark 1. Instead of pushing forward $u_{2}$ from time $t_{k}-$ $\tau$ to time $t_{k}$, one could consider pulling back $v_{2}$ from time

\begin{tabular}{l} 
Step 1: \\
Calculate transition \\
matrices $P_{t_{k}-\tau, \tau}$ \\
and $P_{t_{k}-\tau, 2 \tau}$ \\
\hline Step 2: \\
Calculate coherent \\
pair between $t_{k}-\tau$ \\
and $t_{k}+\tau$
\end{tabular}

FIG. 1. Visualisation of the overlapping window process. $t_{k}+\tau$ to $t_{k}$ to obtain $w_{2}^{\prime}=v_{2} P_{t_{k}, \tau}^{T}$. The vectors $w_{2}$ and $w_{2}^{\prime}$ are similar but because the numerical diffusion generated by the grid is different for the forward and backward (dual) processes, the result is not the same in general.

\section{OCEAN SURFACE FLOW AND LAGRANGIAN CALCULATIONS}

In this section, we describe the input data used to track the evolution of an Agulhas ring and to monitor its decay during its 116-week (December 2, 1998 to February 21, 2001) journey across the South Atlantic.

\section{A. Time-dependent surface flow derived from satellite}

The time-dependent vector fields used to define $\phi$ in this study are given by surface geostrophic currents computed from the elevation of the Sea Surface Height (SSH) referenced by the geoid. In particular, these total SSH fields were obtained by adding Sea Level Anomalies (SLA) measured by altimetry to a mean dynamic topography. ${ }^{27}$ The SLA field was distributed by the Data Unification and Altimeter Combination System (DUACS) available from AVISO (Archiving, Validation and Interpretation of Satellite Oceanographic data). This global product provides a weekly resolution onto a Mercator grid at a spatial resolution of $1 / 3^{\circ}$ in zonal direction, and between $1 / 4^{\circ}$ and $1 / 3^{\circ}$ in meridional direction over the Agulhas region.

\section{B. Selection of initial domain, trajectory integration, and transition matrix calculation}

From a visual analysis of the time-evolution of the SSH field over the South Atlantic, we selected a domain containing a ring-like structure that will be the focus of our investigations. This structure appears to be a large anticyclonic eddy, or Agulhas ring, released from the Agulhas retroflection approximately on 2 nd December 1998 at $34^{\circ} \mathrm{S} / 14^{\circ} \mathrm{E}$.

In our application of Algorithm 2, we chose $t_{1}=$ 30th December 1998, $\tau=$ four weeks, and spaced the $t_{k}$ four weeks apart, $k=1, \ldots, 29$, thus obtaining an estimate of the ring at four-week intervals. The choice of $\tau=$ four weeks is made for a number of reasons. First, the flow time of $2 \tau=$ eight weeks should be sufficiently long for one to observe nontrivial dynamics; the flow map $\phi(t, 2 \tau, \cdot)$ should capture enough nonlinearity to distinguish a ring from Lagrangian trajectory information. Because the altimetry data are output weekly, we need several weeks of flow to achieve this nonlinearity. Second, we cannot flow much longer than eight weeks because the computations quickly become prohibitively expensive. The exponential rate of separation of nearby trajectories means that the number of test points $z_{i, r}$ (see Eq. (4)) required to accurately represent the image box $\phi\left(t, 2 \tau, B_{i}\right)$ grow exponentially quickly $\tau$. In general, the estimates of the rings obtained depend on the length of the finite-time interval $2 \tau$ over which we optimise the coherence or mass transport.

Several algorithms of "automatic eddy detection" based on instantaneous SSH fields exist; for example, Refs. 8 and 23. These algorithms usually return the center of eddies, their 
radius and trajectories over time, and such algorithms could be combined with our approach to produce a more systematic way to successively define the domains of interest. We cover the initial domain by boxes (rectangles), where each box has a side length of $0.044^{\circ}$ longitude and $0.056^{\circ}$ latitude. The side lengths are chosen such that the boxes are approximately square, so that the numerical diffusion produced by the grid is approximately isotropic.

The initial domains for the subsequent time windows $\left[t_{k}-\tau, t_{k}+\tau\right], k=2, \ldots, N$ are chosen manually, using the SSH field as a guide, similar to what was done in step 1, and enclosing the area of interest by a rectangular domain at least twice the size of the suspected ring location, but not many times larger as we wish the computation to focus on the ring. Again, these initial domains are covered by approximately square boxes. On average, we have $m_{k}=7870$ and $n_{k}=11246$ boxes for the initial and final domain, respectively. At time $t_{k}-\tau$, within each box, $169=13^{2}$ Lagrangian particles are uniformly distributed on a finer sub-box grid and advected in the satellite-derived surface flow field with a 4th-order Runge-Kutta integration scheme for a period of 8 weeks, using the Connectivity Modelling System described in Ref. 24. The box covering at time $t_{k}+\tau$ consists of boxes of the same side lengths that cover the terminal points in the 8 -week trajectories.

\section{INVESTIGATION OF THE PATHWAY OF AN AGULHAS RING}

\section{A. Boundaries, pathways, and comparison with other ring diagnostics}

By seeking maximally coherent structures in the Lagrangian fluid flow, our methodology allows the precise detection of the successive position and 2-D shape of the ring. Figure 2 shows the locations of the ring as determined by Algorithm 2 for the period 30th December 1998-21st February 2001.

The ring is initially moving westward for 12 weeks after which it veers northward for another 8 weeks. It then returns on an almost westward pathway, until it reaches the shallow bathymetry of the Walvis Ridge (the south-west to northwest feature around $5^{\circ} \mathrm{E}$ in Figure 2) in early October 1999. After having crossed the Walvis Ridge, the ring continues on a nearly linear westward path across the Mid-Atlantic Ridge (the north to south feature at $15^{\circ} \mathrm{W}$ in Figure 2) and towards the Brazilian coast. The ring in the middle of the first turn northwards (24 Feb 1999) was difficult to estimate well, and we used the averaged push-forward/pull-back procedure described in Remark 1. After February 2001, the ring has decayed so much that it cannot be detected anymore with the technique described here. We ended up with more than 2 years of ring pathway.

Comparing the shape of the ring detected by our method with a visual inspection of the SSH field (Figure 3), we observe a reasonable correspondence between the two diagnostics. The boundaries of the intermediate coherent sets superimposed well with regions of elevated SSH $(\sim 0.75$ to $1.5 \mathrm{~m}$ ). However, contours of elevated SSH are mainly circular along the whole journey of the ring while the boundaries of the coherent sets exhibit complex shapes.

Similar conclusions can be drawn when comparing the boundaries of the coherent sets with another Eulerian diagnostic, the vorticity field (not shown).

\section{B. Decay}

In order to estimate the effective transport of heat, salt, and other tracers of oceanographic and climatic interests from the Indian basin to the South Atlantic Ocean, many authors have focussed their attention on measuring the "decay" of the Agulhas rings. The decay of Agulhas rings has been assessed by measuring over time the strength of the SSH anomalies (cf. Refs. 4, 11, 30, and 32, and references therein), the strength of azimuthal velocities (Refs. 32 and 33 ), the concentration of (numerical) passive tracers, ${ }^{10}$ the magnitude of potential vorticity, ${ }^{35}$ and more recently the water mass characteristics. ${ }^{7}$ These studies usually examine the area of "elevated energy," as measured by SSH or other Eulerian measurement directly derived from the velocity field (e.g., azimuthal velocities or vorticity).

Here, we estimate the decay of a ring by considering the successive structures detected by our method. Since the ring identified at time $t$ loses the least water mass over a 8-week period (centred at time $t$ ), our coherent set approach is well adapted to study water mass transport, in a Lagrangian sense, as opposed to the indirect Eulerian diagnostics mentioned above.

Figure 4 summarises the area and coherence (i.e., the proportion of Lagrangian particles remaining in $\hat{A}_{t_{k}+\tau}$ after starting in $\hat{A}_{t_{k}-\tau}$ over time $2 \tau$ at each windowing step), cf. Figure 1 and Eq. (2).

The area of the box ranges from $24.25 \mathrm{~km}^{2}$ to $27.71 \mathrm{~km}^{2}$, thus our estimates of ring area can only be accurate to this quantum of area, which represents between $0.25 \%$ and $0.04 \%$ of the ring area. This relatively coarse grid introduces numerical diffusion of a diameter between $4.89 \mathrm{~km}$ and $5.66 \mathrm{~km}$ every 8 weeks, which is of the same

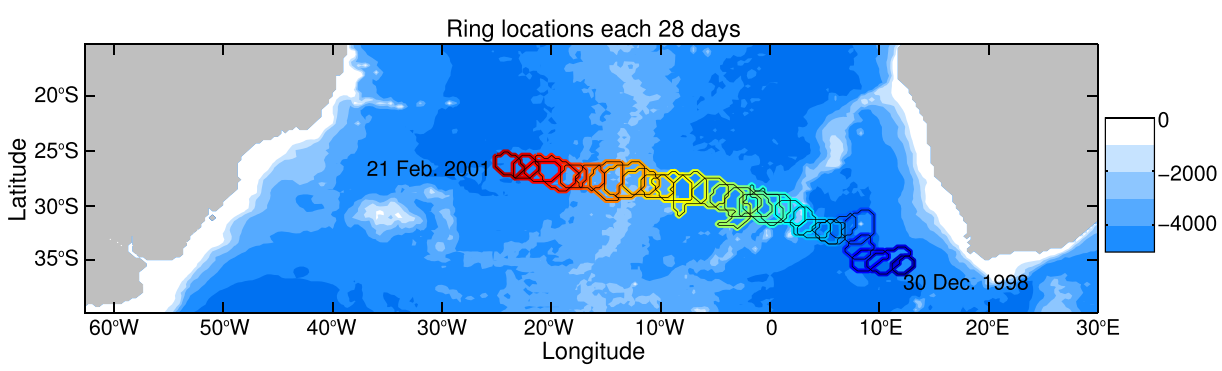

FIG. 2. Successive positions of the Agulhas ring tracked over more than 2 years. The colored elliptic shapes represent the ring boundaries, going from blue through green to red contours as time progresses. Blue shading indicates the main bathymetric features (according to the colorscale). 

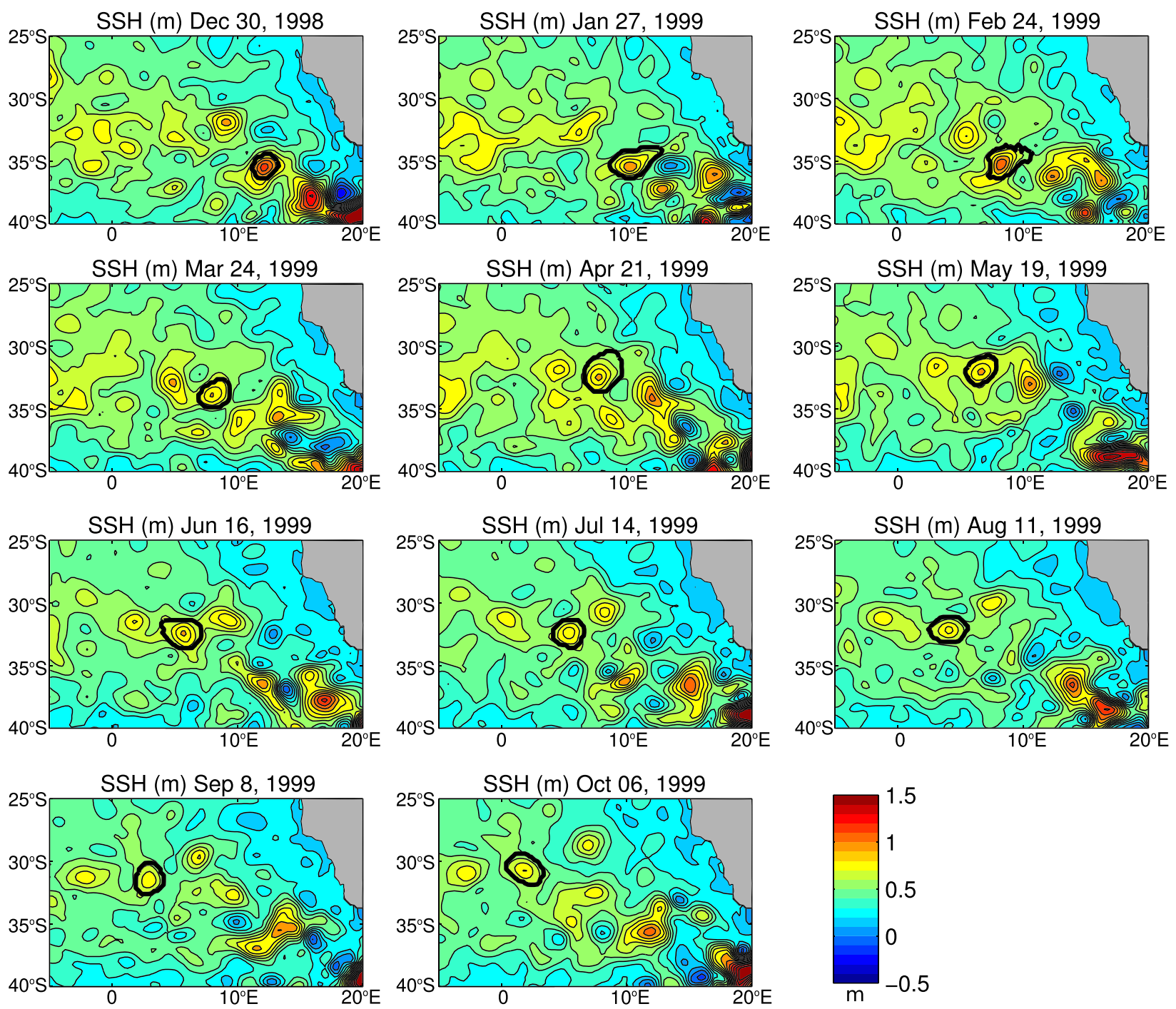

FIG. 3. Sea surface height field during the first 44 weeks of tracking, i.e., from 30 December 1998 to 6 October 1999 , when the ring is still within the Cape Basin. The outputs of Algorithm 2 are overlaid with the thick black curves. Note the good agreement between the tracking method and the SSH field.

order of magnitude of the accepted subgrid-scale diffusion processes.

We found that the 8 -week coherence $\rho\left(\hat{A}_{t_{k}-\tau}, \hat{A}_{t_{k}+\tau}\right)$ associated with each successive ring $\bar{A}_{t_{k}}$ is quite stable and remains very high, a result of our optimized diagnostic. However, the successively detected rings $\bar{A}_{t_{k}}$ have areas that vary substantially from about $19000 \mathrm{~km}^{2}$ up to $100000 \mathrm{~km}^{2}$. While the bottom topography potentially influences ring area (cf. Ref. 20), as does ring decay over time due to energy loss, some of the large fluctuations observed are likely due to a "flat optimum" in the calculation of $\rho$ in Eq. (5). In other words, several contours of the vectors $u_{2}$ and $v_{2}$ achieve very similar values of $\rho$. Dynamically, it suggests that the most coherent ring is ambiguous with several nested rings of different sizes being approximately equally coherent.

Within our framework, a natural way to estimate the decay of the ring in terms of transport (water carried within the ring) is to investigate how many particles remain within the structure detected by our method. For this, we did a forward Runge-Kutta integration (using the same Connectivity Modelling System ${ }^{24}$ and vector fields as used to construct the transition matrix) of 20000 particles initialized within the ring boundary on 30 December 1999. The particles were integrated forward in time for 116 weeks, and their location was recorded every 4 weeks, coinciding with the times at which ring boundaries were available. Then, for each snapshot of ring boundary, the number of particles inside and outside of the ring was counted.

Figure 5 shows the proportion of particles remaining within the ring along its temporal evolution. This has been measured in two ways: for each snapshot, we are (i) counting how many particles are in the ring (green line), i.e., $F_{1}(k)$ $=(\sharp$ particles in ring $k) /(\sharp$ particles in ring 1$)$, and (ii) counting only the particles that have been in the ring in all preceding snapshots (red line), i.e., $F_{2}(k)=(\sharp$ particles in rings $1, \ldots, k) /(\sharp$ particles in ring 1$)$. To compare these Lagrangian diagnostics of decay with a common Eulerian proxy, ${ }^{30}$ the time-evolution of the maximum SSH value observed within the ring is also shown (blue line, Figure 5).

Both lines drop significantly, by more than $10 \%$ between steps 2 and 3 (Feb-Mar 1999), since some particles are expelled from the initial ring in filamentous structures 


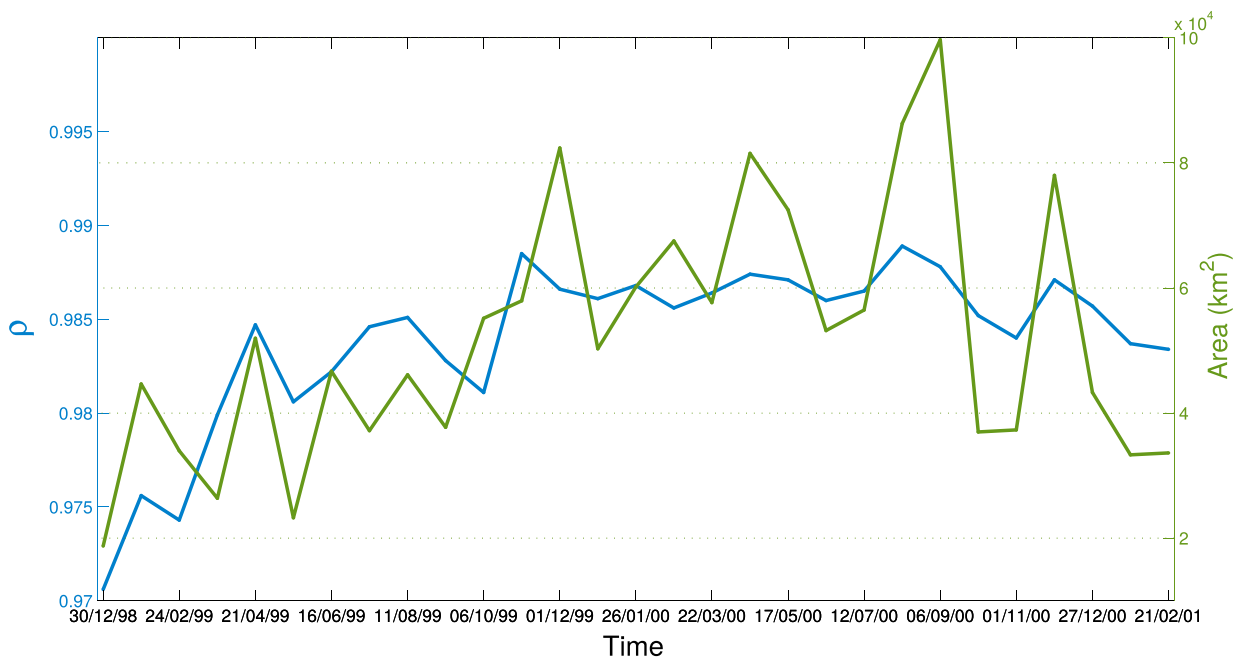

FIG. 4. Coherence and area of the 29 successive positions over the 116weeks period. drifting away from the core of the ring. This fast decay may be due to the ring pathway that made a sudden turn from a westward course to a northward course (see Fig. 2), possibly favouring instabilities and thus the formation of mesoscale filaments. Another possible explanation for this fast decay in Feb-Mar 1999 may be that the time-parameter retained here (i.e., $\tau=4$ weeks) might not be appropriate to capture such fast dynamics. Later, while $F_{2}$ decreases further and remains below 90\% from April 1999, $F_{1}$ rebounds in May 1999 to about $95 \%$ as many of the particles expelled in step 2 were recaptured by the ring. Another significant drop occurs from July to October 1999 in both $F_{1}$ and $F_{2}$. After that date, the amount of particles within the ring slowly decreases, similarly in both proxies $F_{1}$ and $F_{2}$, to about $80 \%-85 \%$ until the end of the 116-week period.

Overall, our analyses suggest that only $15 \%-20 \%$ of the water carried by the ring leaked away from the structure during this 2-year journey. Note that the Eulerian estimate of decay based on SSH reveals a larger temporal variability and suggests a higher decay of about $30 \%$ over the same period.

\section{Discussion and conclusions}

We have proposed a novel approach for the analysis of Agulhas rings based on a sequence of coherent sets and we have successfully applied this method for the tracking of an
Agulhas ring for over 2 years. More precisely, we have demonstrated the existence of a family of rings, indexed fourweekly, which carry a high amount of tracer initialized in the first ring, over a two-year period. Furthermore, we analysed the decay of the ring measured by other methods.

Our technique of performing Lagrangian computations on many short overlapping time intervals was motivated by the desire to allow the coherent sets to vary in size over time. A computational advantage of stitching together a sequence of overlapping short flow times of 8 weeks is that the number of sample points per box can remain relatively low; to achieve a good sampling over the full 116 weeks would require a great deal more tracer particles. The overlapping time window approach proved successful in terms of tracking the location of the ring. It is unclear whether the sometimes rapidly varying size of the computed rings is an artefact of the technique or a reflection of the dynamics in the vector field. It would be possible to include some ad hoc changes to Algorithms 1 and 2 to enforce less variation in ring size. For example, when optimising $\rho$ in (5) one could include a penalty term proportional to the size difference between the current and past ring, or include physically motivated hard constraints on the rate of change of ring area. However, given the ambiguity in the physical definition of the ring boundary, it is not clear whether this is desirable.

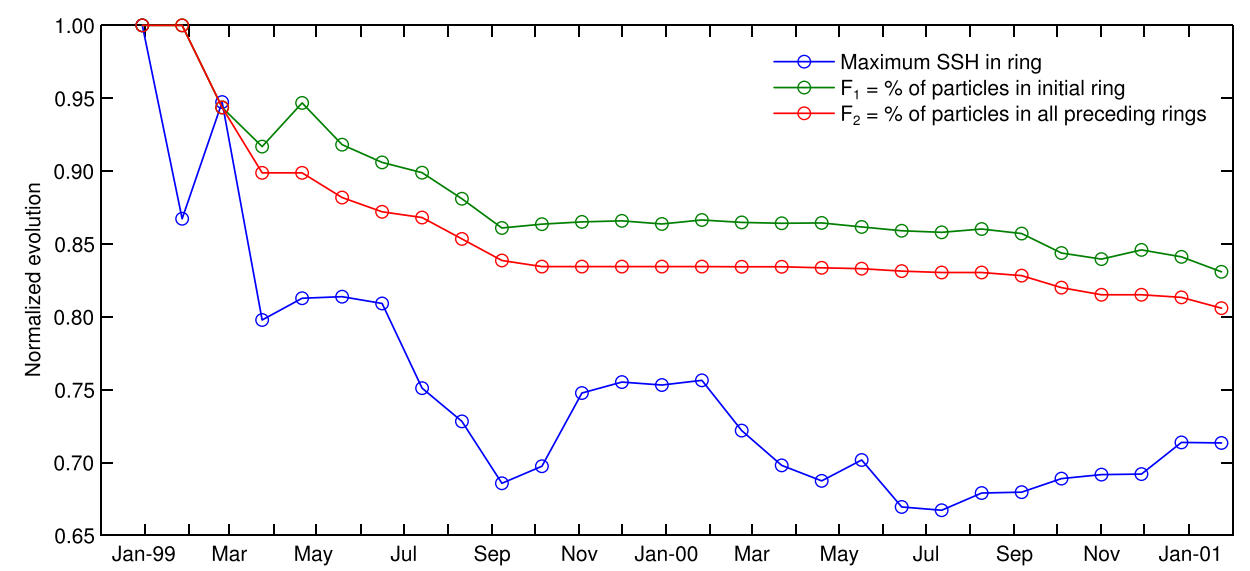

FIG. 5. Time evolution of two Lagrangian proxies of decay of the ring, $F_{1}$ and $F_{2}$, derived from our method versus a simple Eulerian proxy based on SSH. The blue line represents the maximum SSH values within the ring, the green line is the amount of particles in the ring at all snapshots $\left(F_{1}\right)$ and the red line is the number of particles in the ring that have been in the ring on all snapshots before $\left(F_{2}\right)$. All time series are normalised for the ease of comparison. 
Agulhas rings have long been known to carry warm and saline Indian Ocean water into the Southwestern Atlantic Ocean. How much water they carry, however, is under debate. Many studies have documented that Agulhas rings quickly decay in the first 6 months after formation ${ }^{30,32,35}$ and that up to half of the Indian Ocean water that reaches the Atlantic Ocean does so outside Agulhas rings. To better understand the relation between the decay of an Agulhas ring and the amount of water being effectively transported within them, a necessary first step is to accurately identify them in noisy velocity fields. While the recent study of Ref. 2 made progress on this, their assumption of area conservation within the rings meant they were not able to study the decay of the rings. For this reason, the approach followed here is complementary to the geodesic method of Ref. 2; we explicitly allow the ring to change surface (volume) over its lifetime. Moreover, contrasting the study of Ref. 2, we specifically identify the largest possible ring, consistent with a given level of coherence. We also track the ring for a much longer period of time than the computations in Ref. 2, and note that our overlapping window approach could easily be combined with the method of Ref. 2 or other Lagrangian diagnostic methods.

For the case study here, i.e., a typical ring shed in December 1999, we found that approximately $15 \%$ of the Indian Ocean water has leaked from the time-indexed sequence of rings we identified by the time the final ring in the sequence reaches $25^{\circ} \mathrm{W}$ in the western Atlantic Ocean. This is a smaller decay than the ones previously reported by Refs. 30, 32, and 35. Increasing the flow time $2 \tau$ beyond 8 weeks, if it becomes computationally accessible, may lead to slightly different delimitations of the ring for which the decay could be even smaller than reported here because the coherence will be optimised over a longer time duration. Note that another $3 \%$ of the water initially in the ring has been outside of the ring, and later recaptured, along its 116 week lifetime, highlighting how complex the ring evolution is Ref. 4.

There are a few caveats in our study. First, geostrophic currents derived from gridded SSH might not be the most appropriate flow field to accurately estimate the decay of Agulhas rings since, although SSH has a technical resolution of $0.25^{\circ}$, its effective resolution is much lower $\left(\sim 1^{\circ}\right) .^{21}$ To better estimate how remotely observed rings decay, it would be interesting to compare the present calculation against a new altimetric dataset recently reprocessed by Aviso to improve its spatial resolution. Comparing the newer and former products, Ref. 6 found it offers a description of mesoscale activity closer to observations with significantly higher eddy kinetic energy levels, higher eddy densities, and smaller eddy radii. This suggests that both resolution and precision of the velocity field used in our calculation have significant but difficult to predict effects on decay estimates. Going to even smaller spatial scales, geostrophic velocities do not capture the submesoscale processes that are often seen on the flank of larger vortex although they do contribute largely to an effective mixing between the core and the surrounding waters. Second, the oceanic flow is threedimensional in nature and water can move vertically. ${ }^{13}$
However, as in Ref. 2 we here use a two-dimensional altimetric velocity field so that this vertical circulation cannot be resolved.

To study the leakage of tracer out of Agulhas rings over long time period, future work may use high-resolution ocean model to properly consider the effect of these smaller-scale processes in three dimensions. The technique developed here and those described by Refs. 3 and 15 are indeed equally applicable to 3D fields from numerical simulations.

\section{ACKNOWLEDGMENTS}

The altimeter products were produced by Ssalto/Duacs and distributed by Aviso, with support from CNES (http:// www.aviso.oceanobs.com/duacs/). G.F. and C.H. were partially supported by the 2011/12 Go8/DAAD Australia/ Germany Joint Research Co-Operation Scheme. G.F. was also supported by an ARC Future Fellowship. E.v.S. was supported by the ARC via Grant DE130101336. V.R. acknowledges support from MICINN and FEDER through the ESCOLA project (CTM2012-39025-C02-01) while finishing this paper.

${ }^{1}$ L. M. Beal, W. P. M. De Ruijter, A. Biastoch, and R. Zahn, "On the role of the Agulhas system in ocean circulation and climate," Nature 472(7344), 429-436 (2011).

${ }^{2}$ F. J. Beron-Vera, Y. Wang, M. J. Olascoaga, G. J. Goni, and G. Haller, "Objective detection of oceanic eddies and the Agulhas leakage," J. Phys. Oceanogr. 43(7), 1426-1438 (2013).

${ }^{3}$ J. H. Bettencourt, C. López, and E. Hernández-García, "Oceanic threedimensional Lagrangian coherent structures: A study of a mesoscale eddy in the Benguela upwelling region," Ocean Modell. 51, 73-83 (2012).

${ }^{4}$ O. Boebel, J. Lutjeharms, C. Schmid, W. Zenk, T. Rossby, and C. Barron, "The cape cauldron: A regime of turbulent inter-ocean exchange," Deep Sea Res. Part II 50(1), 57-86 (2003).

${ }^{5}$ D. A. Byrne, A. L. Gordon, and W. F. Haxby, "Agulhas eddies: A synoptic view using Geosat erm data," J. Phys. Oceanogr. 25(5), 902-917 (1995).

${ }^{6}$ A. Capet, E. Mason, V. Rossi, C. Troupin, Y. Faugere, I. Pujol, and A. Pascual, "Implications of refined altimetry on estimates of mesoscale activity and eddy-driven offshore transport in the Eastern Boundary Upwelling Systems," Geophys. Res. Lett. 41(21), 7602-7610, doi:10.1002/2014GL061770 (2014).

${ }^{7}$ A. Chaigneau, M. Le Texier, G. Eldin, C. Grados, and O. Pizarro, "Vertical structure of mesoscale eddies in the eastern South Pacific Ocean: A composite analysis from altimetry and Argo profiling floats," J. Geophys. Res. 116(C11), 2156-2202 (2011).

${ }^{8}$ D. B. Chelton, M. G. Schlax, and R. M. Samelson, "Global observations of nonlinear mesoscale eddies," Prog. Oceanogr. 91(2), 167-216 (2011).

${ }^{9}$ W. P. M. de Ruijter, A. Biastoch, S. S. Drijfhout, J. R. E. Lutjeharms, R. P. Matano, T. Pichevin, P. J. van Leeuwen, and W. Weijer, "IndianAtlantic interocean exchange: Dynamics, estimation and impact," J. Geophys. Res.: Oceans 104(C9), 20885-20910 (1999).

${ }^{10}$ L. de Steur, P. J. van Leeuwen, and S. S. Drijfhout, "Tracer leakage from modeled Agulhas rings," J. Phys. Oceanogr. 34(6), 1387-1399 (2004).

${ }^{11}$ G. Dencausse, M. Arhan, and S. Speich, "Routes of Agulhas rings in the southeastern cape basin," Deep Sea Res. Part I 57(11), 1406-1421 (2010).

${ }^{12}$ A. M. Doglioli, M. Veneziani, B. Blanke, S. Speich, and A. Griffa, "A Lagrangian analysis of the Indian-Atlantic interocean exchange in a regional model," Geophys. Res. Lett. 33, L14611, doi:10.1029/ 2006GL026498 (2006).

${ }^{13}$ J. Donners, S. S. Drijfhout, and A. C. Coward, "Impact of cooling on the water mass exchange of Agulhas rings in a high resolution ocean model," Geophys. Res. Lett. 31, L16312, doi:10.1029/2004GL020644 (2004).

${ }^{14} \mathrm{G}$. Froyland, "An analytic framework for identifying finite-time coherent sets in time-dependent dynamical systems," Physica D 250, 1-19 (2013).

${ }^{15}$ G. Froyland, C. Horenkamp, V. Rossi, N. Santitissadeekorn, and A. Sen Gupta, "Three-dimensional characterization and tracking of an Agulhas ring," Ocean Modell. 52-53, 69-75 (2012). 
${ }^{16} \mathrm{G}$. Froyland and K. Padberg-Gehle, "Almost-invariant and finite-time coherent sets: Directionality, duration, and diffusion," in Ergodic Theory, Open Dynamics, and Coherent Structures. Proceedings in Mathematics and Statistics, edited by W. Bahsoun, C. Bose, and G. Froyland (Springer, 2014), Vol. 70, pp. 171-216.

${ }^{17}$ G. Froyland, N. Santitissadeekorn, and A. Monahan, "Transport in timedependent dynamical systems: Finite-time coherent sets," Chaos 20(4), 043116 (2010).

${ }^{18}$ G. J. Goni, S. L. Garzoli, A. J. Roubicek, D. B. Olson, and O. B. Brown, "Agulhas ring dynamics from TOPEX/POSEIDON satellite altimeter data,” J. Mar. Res. 55, 861-883 (1997).

${ }^{19} \mathrm{G}$. Haller and F. J. Beron-Vera, "Geodesic theory of transport barriers in two-dimensional flows," Physica D 241(20), 1680-1702 (2012).

${ }^{20}$ V. M. Kamenkovich, Y. P. Leonov, D. A. Nechaev, D. A. Byrne, and A. L. Gordon, "On the influence of bottom topography on the Agulhas eddy," J. Phys. Oceanogr. 26(6), 892-912 (1996).

${ }^{21}$ S. R. Keating, K. S. Smith, and P. R. Kramer, "Diagnosing lateral mixing in the upper ocean with virtual tracers: Spatial and temporal resolution dependence,” J. Phys. Oceanogr. 41, 1512-1534 (2011).

${ }^{22}$ C. Mendoza, A. M. Mancho, and S. Wiggins, "Lagrangian descriptors and the assessment of the predictive capacity of oceanic data sets," Nonlinear Processes Geophys. 21(3), 677-689 (2014).

${ }^{23}$ F. Nencioli, C. Dong, T. Dickey, L. Washburn, and J. C. McWilliams, "A vector geometry-based eddy detection algorithm and its application to a high-resolution numerical model product and high-frequency radar surface velocities in the Southern California Bight," J. Atmos. Oceanic Technol. 27(3), 564-579 (2010).

${ }^{24}$ C. B. Paris, J. Helgers, E. van Sebille, and A. Srinivasan, "Connectivity modeling system: A probabilistic modeling tool for the multi-scale tracking of biotic and abiotic variability in the ocean," Environ. Modell. Software 42(0), 47-54 (2013).
${ }^{25}$ J. B. Pesin, "Families of invariant manifolds corresponding to nonzero characteristic exponents," Math. USSR-Izv. 10(6), 1261 (1976).

${ }^{26}$ C. Pugh and M. Shub, "Ergodic attractors," Trans. Am. Math. Soc. 312(1), 1-54 (1989).

${ }^{27}$ M.-H. Rio, S. Guinehut, and G. Larnicol, "New CNES-CLS09 global mean dynamic topography computed from the combination of grace data, altimetry, and in situ measurements," J. Geophys. Res.: Oceans 116, C07018 (2011).

${ }^{28}$ V. Rom-Kedar and S. Wiggins, "Transport in two-dimensional maps," Arch. Ration. Mech. Anal. 109(3), 239-298 (1990).

${ }^{29}$ I. I. Rypina, S. E. Scott, L. J. Pratt, and M. G. Brown, "Investigating the connection between complexity of isolated trajectories and Lagrangian coherent structures," Nonlinear Processes Geophys. 18, 977-987 (2011).

${ }^{30}$ M. W. Schouten, W. P. M. de Ruijter, P. J. van Leeuwen, and J. R. E. Lutjeharms, "Translation, decay and splitting of Agulhas rings in the southeastern Atlantic Ocean,” J. Geophys. Res. 105(C9), 21913-21925, doi:10.1029/1999JC000046 (2000).

${ }^{31}$ S. C. Shadden, F. Lekien, and J. E. Marsden, "Definition and properties of Lagrangian coherent structures from finite-time Lyapunov exponents in two-dimensional aperiodic flows," Physica D 212, 270-304 (2005).

${ }^{32}$ A. M. Treguier, O. Boebel, B. Barnier, and G. Madec, "Agulhas eddy fluxes in a 1/6 Atlantic model," Deep Sea Res. Part II 50(1), 251-280 (2003).

${ }^{33}$ H. M. van Aken, A. K. van Veldhoven, C. Veth, W. P. M. de Ruijter, P. J. van Leeuwen, S. S. Drijfhout, C. P. Whittle, and M. Rouault, "Observations of a young Agulhas ring, astrid, during mare in march 2000," Deep Sea Res. Part II 50(1), 167-195 (2003).

${ }^{34}$ P. J. van Leeuwen, "The propagation mechanism of a vortex on the plane," J. Phys. Oceanogr. 37(9), 2316-2330 (2007).

${ }^{35}$ E. van Sebille, P. J. van Leeuwen, A. Biastoch, and W. P. M. de Ruijter, "On the fast decay of Agulhas rings," J. Geophys. Res. 115, C03010 (2010). 